\title{
Atuação do enfermeiro na segurança do paciente em centro cirúrgico: revisão integrativa da literatura
}

\author{
Nursing actions in patient safety at a surgical center: integrative literature review \\ actuación del enfermero en la seguridad del paciente en centro quirúrgico: revisión \\ integrativa de la literatura
}

Thalyta Mariany Rêgo Lopes ${ }^{1 *}$, Alessandra Valeska Araujo Machado¹, Aurea Silva da Silva ${ }^{1}$, Terezinha de Jesus Xavier dos Santos ${ }^{1}$, lanny Ferreira Raiol ${ }^{1}$, Shirley Aviz de Miranda ${ }^{1}$, Juliana Conceição Dias Garcez ${ }^{1}$, Paula Sousa da Silva Rocha².

\section{RESUMO}

Objetivo: Analisar as tendências das produções científicas acerca da atuação do enfermeiro na segurança do paciente em centro cirúrgico no período de 2013 a 2017. Métodos: Trata-se de uma Revisão Integrativa da Literatura. Foram utilizadas como critérios de inclusão artigos publicados no período de 2013 a 2017; textos completos em português, resultando em 19 artigos. Para análise dos dados utilizou-se a técnica de Bardin. Resultados: O maior número de publicações ocorreu no ano de $2017 \mathrm{com}$ (6) produções cientificas, e tem como base de dados mais frequente a LILACS com (10), foram identificados (8) artigos de abordagem quantitativa, (9) qualitativa e (2) quali-quantitativa. Na temática formou-se três categorias: Eventos adversos ocorridos em centro cirúrgicos relacionados a segurança do paciente; Implantação do instrumento de checagem na segurança do paciente (checklist); Realização, treinamento, comprometimentos e notificação de ocorrência da equipe de Enfermagem do centro cirúrgico. Conclusão: A análise permitiu ressaltar que é uma temática bastante discutida atualmente, entretanto é necessário realizar capacitações aos profissionais sobre métodos de como conduzir e melhorar a qualidade da assistência prestada por meio principalmente, de prevenção.

Palavras-chave: Segurança do Paciente, Centro Cirúrgico, Enfermagem de centro cirúrgico.

\begin{abstract}
Objective: To analyze the trends of scientific production about the role of nurses in patient safety in a surgical center from 2013 to 2017. Methods: Type Integrative Literature Review. The articles inclusion criteria were published research from 2013 to 2017; only were selected papers in portuguese, resulting in 19 articles. For data analysis, the Bardin technique was used. Results: The greatest number of publications occurred in 2017 with (6) scientific productions, and has as a more frequent database LILACS with (10), were identified (8) articles of quantitative, (9) qualitative and 2) qualitative and quantitative, three categories were formed: Adverse events occurred in surgical centers, related to patient safety; Implantation of the instrument of check in the patient's safety (checklist); Realization, training, commitments and notification of occurrence of the nursing team of the surgical center. Conclusion: The analysis made it possible to emphasize that it is a topic that is much discussed at the moment, it is necessary to provide training to professionals on methods of conducting and improving the quality of care provided through prevention.
\end{abstract}

Key words: Patient Safety, Surgical Center, Surgical Center Nursing.

${ }^{1}$ Centro Universitário Metropolitano da Amazônia (UNIFAMAZ), Belém-Pará. *E-mail: thalyta_mlopes@hotmail.com

${ }^{2}$ Centro Universitário do Estado do Pará (CESUPA), Belém - Pará. 


\section{RESUMEN}

Objetivo: Analizar las tendencias de las producciones científicas cerca de la actuación del enfermero en la seguridad del paciente en centro quirúrgico en el período de 2013 a 2017. Métodos: investigación cualitativa descriptiva del tipo Revisión Integrativa de la Literatura. Se utilizaron las bases de datos electrónicas. Se seleccionaron artículos publicados en el período de 2013 a 2017; texto completo en portugués, lo que resulta en 19 artículos. Para el análisis de los datos se utilizó la técnica de Bardin. Resultados: El mayor número de publicaciones ocurrió en el año 2017 con (6) producciones científicas y tiene como base de datos más frecuente a LILACS con (10), se identificaron (8) artículos de abordaje cuantitativo, (9) cualitativo y () 2) cualcuantitativa. En la temática se formaron tres categorías: Eventos adversos ocurridos en centro quirúrgicos relacionados a la seguridad del paciente; Implantación del instrumento de chequeo en la seguridad del paciente (checklist); Realización, entrenamiento, comprometimiento y notificación de ocurrencia del equipo de Enfermería del centro quirúrgico. Conclusión: El análisis permitió resaltar que es una temática bastante discutida actualmente, además es necesario realizar capacitaciones a los profesionales sobre métodos de cómo conducir y mejorar la calidad de la asistencia prestada por medio principalmente de prevención.

Palabras clave: Seguridad del paciente, Centro quirúrgico, Enfermería de centro quirúrgico.

\section{INTRODUÇÃO}

Avanços científicos e tecnológicos propiciam um aumento no número de procedimentos, inclusive de intervenções cirúrgicas. Também são crescentes as iniciativas para a promoção da segurança e da qualidade na assistência à saúde em âmbito mundial, com envolvimento da alta direção das instituições até seus colaboradores (OLIVEIRA RM, et al., 2014).

E nessa última década a preocupação com a segurança do paciente tornou-se constante para todo o setor de saúde em todas as suas dimensões, principalmente, em um ambiente no qual se desenvolvam atividades complexas, interdisciplinares e com forte dependência da atuação individual; em um ambiente de alto risco para a ocorrência de eventos adversos, como o ambiente do centro cirúrgico (OLIVEIRA RM, et al., 2014).

Nessa perspectiva, busca-se com a segurança do paciente a redução e a diminuição de atos considerados inseguros atrelados ao sistema de assistência à saúde, com emprego das melhores práticas no intuito de obter os resultados esperados (SECANELL M, 2014).

Sendo assim, com o intuito de eliminar e reduzir os riscos aos pacientes a Organização Mundial de Saúde (OMS), em 2004, lançou a Aliança Mundial para Segurança do Paciente com o objetivo melhorar a segurança do cuidado, desenvolvendo política e estratégia na atenção à saúde (PANCIERI AP, et al., 2013).

De acordo com Bezerra WR et al. (2015) de 750 internações relacionados a procedimentos cirúrgicos, a maioria apresenta dor aguda no pós-operatório, dispositivos tubulares retirados precipitadamente, reações adversas medicamentosas, infecções hospitalares e falha em procedimentos técnicos.

É notório que o aumento no número de cirurgias ocorreu devido ao avanço tecnológico e isso trouxe benefícios consideráveis para os pacientes. Procedimentos cirúrgicos complexos se tornam rotineiros, mas ao mesmo tempo, deixaram o ambiente cirúrgico mais inseguro (GOMES JAP, et al., 2016).

Diante disso, no ambiente do centro cirúrgico entre 2007 e 2008, foi implantado o protocolo da cirurgia segura, com o intuito de reforçar as práticas de segurança cirúrgica estabelecidas pela OMS. Esse protocolo é importante para a segurança ao paciente cirúrgico e passam a ser aplicados em todos os países membros da OMS (PANCIERI AP, et al., 2013).

Nesse sentido, entende-se que o centro cirúrgico é um ambiente avolumado de tarefas complexas, exigese um profissional que tenha atenção redobrada em todos os processos, dentre esses, o enfermeiro tem papel primordial devido suas ações gerenciais e assistências prestadas ao paciente, como a realização do checklist (LIMA AM, et al., 2013). 
O enfermeiro é essencial na segurança do paciente no centro cirúrgico, assim, contribuindo para prevenção de erros, visando à recuperação do paciente através de suas intervenções eficazes e técnicas, devido ao desenvolvimento de práticas essenciais à vida e estabelecendo um diálogo entre pacientes e familiares, esclarecendo acerca dos procedimentos cirúrgicos estabelecidos e incluindo os cuidados na cirurgia segura (SALBEGO C, et al., 2015)

Além disso, durante as atividades práticas desenvolvidas pelas acadêmicas de enfermagem no centro cirúrgico despertou-se o interesse de entender sobre o gerenciamento de risco e o Programa de Segurança do Paciente (Cirurgia Segura) e suas propostas de melhoria na assistência.

Assim, a contribuição desse trabalho é entender como se dá atuação do enfermeiro na segurança do paciente em ambiente cirúrgico, além de despertar nos enfermeiros e acadêmicos de enfermagem a importância de conhecer os protocolos que visem à segurança do paciente em centro cirúrgico e, dessa forma, garantir a assistência de enfermagem livre de danos e riscos.

Diante do exposto, da importância e complexidade do tema, além de perceber o enfermeiro como peça primordial para a garantia da segurança do paciente em centro cirúrgico, a questão que impulsiona esse estudo por meio de uma revisão integrativa de literatura: Quais as evidências científicas sobre a atuação do enfermeiro na segurança do paciente no centro cirúrgico, no período de 2013 a 2017 ?

A partir das atualizações que vem sendo realizadas no âmbito profissional que visam a segurança do paciente e a preocupação com a cirurgia segura. O presente estudo teve por objetivo, analisar a tendência das produções científicas, acerca da atuação do enfermeiro na segurança do paciente em centro cirúrgico no período de 2013 a 2017.

\section{MÉTODOS}

O presente estudo trata-se de uma Revisão Integrativa da Literatura (RIL), que consiste em um estudo descritivo, com abordagem qualitativa. É um método específico que possui a finalidade de reunir e sintetizar resultados de pesquisa anteriores de maneira sistemática e ordenada, contribuindo assim, para maior aprofundamento do tema já investigado. Objetiva-se com esse método de revisão apontar lacunas do conhecimento, que precisam ser preenchidas e a necessidade da realização de novos estudos (SILVA JL, et al., 2015).

Para o desenvolvimento da Revisão integrativa, foram realizadas as seguintes etapas, primeiramente a elaboração das perguntas norteadoras; depois foi estabelecido os critérios de inclusão e de exclusão; na sequência foram definidas as informações a serem extraídas dos artigos selecionados; após foi realizado a avaliação dos estudos incluídos; e por fim foi realizado as interpretações dos resultados e a apresentação da revisão integrativa.

Para melhor indicar a direção da pesquisa foi elaborada a seguinte pergunta norteadora: Quais as evidências científicas sobre a atuação do enfermeiro na segurança do paciente no centro cirúrgico, no período de 2013 a $2017 ?$

A pesquisa foi desenvolvida no período de fevereiro a abril de 2018 por meio de busca científica, foram utilizadas as bases de dados da Biblioteca Virtual da Saúde (BVS), da Literatura Latina Americana e do Caribe em Ciências da Saúde (LILACS), Medical Literature Analysis and Retrieval Sistem (MEDLINE), Base de dados de Bibliográficas Especializada na área de Enfermagem (BDENF). Na busca dos artigos utilizou-se os descritores: "Cuidado de Enfermagem", "Segurança do Paciente", "Enfermagem de centro cirúrgico". Foi realizado a busca com descritores fazendo o cruzamento com o conector AND no campo de descritores na Biblioteca Virtual da Saúde.

Para a qualidade de critério de inclusão na pesquisa foram utilizados textos completos online, língua portuguesa, artigos publicados no período de 2013 a 2017, cujas publicações estavam nas bases e utilizados os descritores propostos para a busca científica. Os Critérios de exclusão foram os artigos que estavam 
repetidos nas bases de dados, fora do período de revisão bibliográfica, que não eram em língua portuguesa, dissertações e teses. Após a busca eletrônica foi realizada a pré-seleção pelos pesquisadores, feito uma leitura minuciosa dos títulos e resumos dos artigos com a intenção de que esteja conforme os critérios de inclusão estabelecidos. Foram achados no primeiro momento 361 artigos, porém, após a leitura relacionada à pergunta norteadora utilizou-se 19 dessas publicações. Portanto, depois da avaliação de levantamentos dos artigos, esses foram analisados a um instrumento adaptado da URSI 2005; este instrumento é composto por vários eixos de avaliação metodológica, no entanto foi adaptado para esta pesquisa, para apenas dois eixos e suas respectivas subdivisões, a primeira: Eixo 1 Perfil das produções, e a segunda: Eixo 2 Resultados em evidência.

A análise dos dados foi realizada por meio da técnica de análise de conteúdo de Bardin, no qual caracteriza-se como métodos e técnicas específicas para elaboração esquemática, para compreender os sentidos das comunicações e suas especificações. Dentre esses foram usados as seguintes fases: descrição dos dados de identificação das publicações (autores e ano), do Estado e Instituição, sede do estudo e do tipo de revista científica; em seguida organizados em quadro; foi descrita as características metodológicas dos estudos, classificando-os de acordo com o delineamento de pesquisa e nível de evidências; descrição da análise propriamente dita, agrupadas em categorias (SILVA AH; FOSSÁ MIT, 2017).

\section{RESULTADOS}

O quadro 1 representa pesquisas utilizadas nas bases de dados conforme critérios de inclusão. No estudo incluiu-se 19 evidências científicas, utilizando um instrumento adaptado da URSI (2005). Nesta tabela usouse caracterização contendo algumas informações como: título do artigo, autores, ano, periódico, base de dados, método e resumo dos dados encontrados.

O maior número de publicações ocorreu no ano de 2017 com 06 (31,6\%) produções científicas, e tem como base de dados mais frequente a LILACS com 10 (52\%) artigos. As evidências científicas foram distribuídas em 8 periódicos, entres eles: Revista Gaúcha (2), ACTA Paulista de Enfermagem (3), Revista SOBECC Associação Brasileira de Enfermeiro de Centro Cirúrgico Anestésica e Centro de Material e Esterilização (4), Revista de Enfermagem UFPI (1), Revista de Enfermagem UFPE (2), Revista e Escola de Enfermagem USP (1), Cogitare Enfermagem UFPR (5), Revista de Enfermagem online (1).

Relacionado à pesquisa metodológica, foi identificado que 8 (42,1\%) são de abordagem Quantitativo e 9 (47,3\%) qualitativa e $2(42,1 \%)$ Quali-quantitativa.

Realizou-se análise qualitativa baseadas em resultados evidenciados e, encontrados três categorias, como: Eventos adversos ocorridos em centro cirúrgicos; Implantação do instrumento de checagem na segurança do paciente (checklist); Realização, treinamento, comprometimento e notificação de ocorrência da equipe de Enfermagem do centro cirúrgico.

$\mathrm{Na}$ primeira categoria identificou-se 5 artigos que ressaltam eventos adversos ocorridos em centro cirúrgico relacionando-se com a falta de comunicação efetiva entre os profissionais, falta de demarcação da lateralidade cirúrgica, falta de orientação quanto ao jejum, alergia não relatada, posição cirúrgica, efeitos anestésicos pós cirúrgicos, identificação incorreta do paciente, prontuário incorreto, mais a sobre-carga de trabalho da equipe do centro cirúrgico e a falta da conferência de materiais cirúrgicos.

Na segunda categoria encontra-se 5 artigos que demostraram tradução, construção e a implantação do teste de segurança para avaliar a equipe do centro cirúrgico e a segurança do paciente, com um sistema de fácil preenchimento e resultado eficaz.

$\mathrm{Na}$ terceira categoria destacam-se 9 artigos que evidenciaram a importância da realização de treinamentos, comprometimentos dos profissionais de saúde e notificações conforme orientações estabelecidas pela OMS, resultando assim, na segurança dos profissionais de enfermagem em aplicar corretamente e com qualidade os instrumentos para a segurança do usuário do centro cirúrgico. 
Quadro 1- Caracterização dos estudos quanto ao número de ordem, títulos, autores, ano, periódico, base de dados, resumo, 2018.

\begin{tabular}{|c|c|c|c|c|c|c|c|}
\hline $\mathbf{N}^{\circ}$ & TÍTULO & AUTOR(S) & ANO & PERIÓDICO & BD & METÓDO & RESUMO \\
\hline 01 & $\begin{array}{l}\text { Eventos adversos em } \\
\text { pacientes cirúrgicos: } \\
\text { conhecimentos dos } \\
\text { profissionais de } \\
\text { enfermagem }\end{array}$ & $\begin{array}{l}\text { BOHOMOL } \\
\text { E, TARTALI } \\
\text { JA }\end{array}$ & 2013 & $\begin{array}{l}\text { Rev. Acta. } \\
\text { Paul de } \\
\text { Enfermagem }\end{array}$ & LILACS & $\begin{array}{l}\text { Estudo } \\
\text { quantitativo }\end{array}$ & $\begin{array}{l}\text { As rotinas na programação de procedimentos eletivos } \\
\text { e de comunicações não estão sendo favoráveis a um } \\
\text { resultado positivo, a não conferência e identificação } \\
\text { dos pacientes e dos materiais e equipamentos } \\
\text { utilizados, a falta de identificação do profissional } \\
\text { responsável é bem significativa. Ocasionando } \\
\text { eventos adversos. }\end{array}$ \\
\hline 02 & $\begin{array}{l}\text { Avaliação da cultura de } \\
\text { segurança do paciente } \\
\text { em Centro Cirúrgico }\end{array}$ & $\begin{array}{l}\text { CORREGI } \\
\text { O TC, et al. }\end{array}$ & 2014 & Rev. Sobecc. & BDENF & $\begin{array}{l}\text { Estudo } \\
\text { quantitativo }\end{array}$ & $\begin{array}{l}\text { Teste efetuado para avaliar equipe de centro cirúrgico } \\
\text { em relação a comunicação, stress e, atividades de } \\
\text { trabalho, educação continuada oferece melhoria e } \\
\text { adequação para segurança do paciente }\end{array}$ \\
\hline 03 & $\begin{array}{l}\text { Cirurgias seguras: } \\
\text { elaboração de um } \\
\text { instrumento } \\
\text { enfermagem } \\
\text { perioperatório }\end{array}$ & $\begin{array}{l}\text { MONTEIR } \\
\text { O EL, et al. }\end{array}$ & 2014 & Rev. Sobecc & BDENF & $\begin{array}{l}\text { Abordagem } \\
\text { qualitativa }\end{array}$ & $\begin{array}{l}\text { Implantação de instrumento que avalia segurança do } \\
\text { paciente com informações individuais, desde } \\
\text { anamnese a diagnóstico de enfermagem, e } \\
\text { procedimentos certos, sendo assim, uma nova } \\
\text { perspectiva na avaliação de enfermagem para cirurgia } \\
\text { segura. }\end{array}$ \\
\hline 04 & $\begin{array}{l}\text { Validação de checklist } \\
\text { cirúrgico para prevenção } \\
\text { de infecção de sítio } \\
\text { cirúrgico }\end{array}$ & $\begin{array}{l}\text { ROSCANI } \\
\text { ANCP et al. }\end{array}$ & 2015 & $\begin{array}{l}\text { ACTA Paul } \\
\text { Enfermagem }\end{array}$ & LILACS & $\begin{array}{l}\text { Estudo } \\
\text { quantitativo }\end{array}$ & $\begin{array}{l}\text { Aplicar conjuntamente as etapas de verificação de } \\
\text { segurança, assim previne-se risco de infecção do sítio } \\
\text { cirúrgico, esse instrumento usado adequadamente } \\
\text { tem eficácia, intervenção evitando permanência } \\
\text { hospitalar. }\end{array}$ \\
\hline
\end{tabular}




\begin{tabular}{|c|c|c|c|c|c|c|c|}
\hline 05 & $\begin{array}{l}\text { Análise de eventos } \\
\text { adversos em um centro } \\
\text { cirúrgico ambulatorial }\end{array}$ & $\begin{array}{l}\text { SILVA FG, } \\
\text { et al }\end{array}$ & 2015 & $\begin{array}{l}\text { Rev. } \\
\text { SOBECC }\end{array}$ & LILACS & $\begin{array}{l}\text { Estudo } \\
\text { quantitativo }\end{array}$ & $\begin{array}{l}\text { O comprometimento do profissional de notificar um } \\
\text { evento ocorrido é sua responsabilidade, garantido } \\
\text { assim, a segurança do paciente; para que isso ocorra } \\
\text { se faz necessário a capacitação do trabalhador, } \\
\text { envolvendo-o nesse processo assistencial, o qual } \\
\text { vem caracterizar um melhor resultado da assistência. }\end{array}$ \\
\hline 06 & $\begin{array}{l}\text { Adesão ao uso de } \\
\text { checklist cirúrgico para } \\
\text { segurança do paciente }\end{array}$ & $\begin{array}{l}\text { MAZIERO } \\
\text { ECS et al }\end{array}$ & 2015 & $\begin{array}{l}\text { Rev. Gaúcha } \\
\text { de } \\
\text { Enfermagem }\end{array}$ & LILACS & Estudo qualitativo & $\begin{array}{l}\text { Há a existência do protocolo de segurança no centro } \\
\text { cirúrgico, porém eles não são aplicados } \\
\text { continuamente-o que pode levar a eventos adversos }\end{array}$ \\
\hline 07 & $\begin{array}{l}\text { Gerenciamento da } \\
\text { segurança do paciente } \\
\text { sob a ótica dos } \\
\text { enfermeiros }\end{array}$ & $\begin{array}{l}\text { FRANÇOLI } \\
\mathrm{N} \text { L, et al }\end{array}$ & 2015 & $\begin{array}{l}\text { Rev. Esc. } \\
\text { Enfermagem } \\
\text { USP }\end{array}$ & MEDLINE & $\begin{array}{l}\text { Estudo } \\
\text { quantitativo }\end{array}$ & $\begin{array}{l}\text { Há evidência da existência das ações sendo } \\
\text { desenvolvidas em instituições hospitalares, pautadas } \\
\text { em gerenciamentos da segurança do paciente pelo } \\
\text { enfermeiro e sua equipe, sendo o facilitador do } \\
\text { processo de identificação dos riscos. }\end{array}$ \\
\hline 08 & $\begin{array}{l}\text { Produção científica da } \\
\text { Enfermagem de centro } \\
\text { cirúrgico de } 2003 \text { a } 2013\end{array}$ & $\begin{array}{l}\text { CAMPOS } \\
\text { JAR et al }\end{array}$ & 2015 & $\begin{array}{l}\text { Rev. } \\
\text { SOBECC }\end{array}$ & BDENF & $\begin{array}{l}\text { Abordagem } \\
\text { quanti-qualitativa }\end{array}$ & $\begin{array}{l}\text { Evidenciada quantidade reduzida de artigos } \\
\text { relacionados a assistência à criança, adolescente e } \\
\text { idoso; os quais poderiam ser explorados para um } \\
\text { planejamento da assistência de Enfermagem no } \\
\text { centro cirúrgico. }\end{array}$ \\
\hline 09 & $\begin{array}{l}\text { Segurança do paciente } \\
\text { no ambiente cirúrgico: } \\
\text { tradução e adaptação } \\
\text { cultural de instrumento } \\
\text { validado }\end{array}$ & $\begin{array}{l}\text { LOURENÇ } \\
\tilde{A} O \text { D CA, } \\
\text { TRONCHIN } \\
\text { DMR }\end{array}$ & 2016 & $\begin{array}{l}\text { Rev. Acta } \\
\text { Paul } \\
\text { Enfermagem }\end{array}$ & BDENF & $\begin{array}{l}\text { Abordagem } \\
\text { quanti-qualitativa }\end{array}$ & $\begin{array}{l}\text { Realização de tradução e adaptação para realidade } \\
\text { brasileira de dois formulários que estavam sendo } \\
\text { aplicados em vários países para mensurar o clima de } \\
\text { segurança dos profissionais de saúde que atuam em } \\
\text { centro cirúrgico }\end{array}$ \\
\hline
\end{tabular}




\begin{tabular}{|c|c|c|c|c|c|c|c|}
\hline 10 & $\begin{array}{l}\text { Processo de contagem } \\
\text { cirúrgica: Evidências } \\
\text { para a segurança do } \\
\text { paciente }\end{array}$ & $\begin{array}{l}\text { FREITAS } \\
\text { PS; et al }\end{array}$ & 2016 & $\begin{array}{l}\text { Rev. Gaúcha } \\
\text { de } \\
\text { Enfermagem }\end{array}$ & BDENF & $\begin{array}{l}\text { Estudo } \\
\text { quantitativo }\end{array}$ & $\begin{array}{l}\text { Para uma cirurgia segura os enfermeiros devem } \\
\text { seguir a Organização Mundial de Saúde que } \\
\text { preconize a padronização dos materiais em qualquer } \\
\text { cirurgia, assim, evitando erros da equipe. }\end{array}$ \\
\hline 11 & $\begin{array}{l}\text { Instrumentos } \\
\text { avaliar a qualidade de } \\
\text { segurança no bloco } \\
\text { operatório- } \\
\text { integrativa }\end{array}$ & $\begin{array}{l}\text { GOMES } \\
\text { JAP et al. }\end{array}$ & 2016 & $\begin{array}{l}\text { Rev. } \\
\text { Cogitare } \\
\text { Enfermagem } \\
\text { UFPR }\end{array}$ & LILACS & $\begin{array}{l}\text { Abordagem } \\
\text { qualitativa }\end{array}$ & $\begin{array}{l}\text { Vem colaborar positivamente, já que o centro cirúrgico } \\
\text { é o local no qual o paciente está mais vulnerável a } \\
\text { qualquer tipo de erro que possam lhe causar dano; } \\
\text { logo, a introdução de técnicas modernas, modelos de } \\
\text { gestão e aplicação de novos instrumentos garantes } \\
\text { qualidade e segurança do paciente. }\end{array}$ \\
\hline 12 & $\begin{array}{l}\text { significado da } \\
\text { segurança do paciente } \\
\text { cirúrgico pediátrico para } \\
\text { a equipe de enfermagem }\end{array}$ & $\begin{array}{l}\text { REIS AT et } \\
\text { al. }\end{array}$ & 2016 & $\begin{array}{l}\text { Rev. } \\
\text { Cogitare } \\
\text { Enfermagem } \\
\text { UFPR }\end{array}$ & LILACS & $\begin{array}{l}\text { Abordagem } \\
\text { qualitativa }\end{array}$ & $\begin{array}{l}\text { O interesse da equipe } \\
\text { de enfermagem em melhorar os serviços prestados } \\
\text { nas cirurgias pediátricas, evidenciando, porém, há } \\
\text { necessidade de empenho em executar o uso dos } \\
\text { protocolos. }\end{array}$ \\
\hline 13 & $\begin{array}{l}\text { Assistência de } \\
\text { enfermagem na } \\
\text { segurança do paciente: } \\
\text { Revisão integrativa }\end{array}$ & $\begin{array}{l}\text { HENRIQUE } \\
S \text { AHB, et } \\
\text { al. }\end{array}$ & 2016 & $\begin{array}{l}\text { Rev. } \\
\text { Cogitare } \\
\text { Enfermagem } \\
\text { UFPR }\end{array}$ & LILACS & $\begin{array}{l}\text { Abordagem } \\
\text { qualitativa }\end{array}$ & $\begin{array}{l}\text { A implantação da atividade educativa com o paciente } \\
\text { e funcionário, sobre segurança do paciente em centro } \\
\text { cirúrgico, executada pelo enfermeiro ocasiona } \\
\text { melhorias gradativas a qualidade da assistência, } \\
\text { antes, durante e depois da cirurgia. }\end{array}$ \\
\hline 14 & $\begin{array}{l}\text { Teste piloto de checklist } \\
\text { de cirurgia segura: relato } \\
\text { de experiência }\end{array}$ & $\begin{array}{l}\text { SANTOS } \\
\text { JS et al. }\end{array}$ & 2017 & $\begin{array}{l}\text { Rev. de } \\
\text { Enfermagem } \\
\text { UFPI }\end{array}$ & MEDLINE & $\begin{array}{l}\text { Abordagem } \\
\text { qualitativa }\end{array}$ & $\begin{array}{l}\text { O checklist tem que ser um formulário de fácil } \\
\text { aplicação com comprometimento da equipe em } \\
\text { aplicá-lo como uma cultura de segurança, uma } \\
\text { política de educação permanente - o que é primordial. }\end{array}$ \\
\hline
\end{tabular}




\begin{tabular}{|c|c|c|c|c|c|c|c|}
\hline 15 & $\begin{array}{l}\text { Construção de manual } \\
\text { sobre cirurgia segura } \\
\text { para profissionais de } \\
\text { saúde }\end{array}$ & $\begin{array}{l}\text { SOUZA } \\
\text { GSL, } \\
\text { RIBEIRO } \\
\text { MRR }\end{array}$ & 2017 & $\begin{array}{l}\text { Rev. } \\
\text { Cogitare } \\
\text { Enfermagem } \\
\text { UFPR }\end{array}$ & LILACS & $\begin{array}{l}\text { Abordagem } \\
\text { qualitativa do tipo } \\
\text { estudo de caso }\end{array}$ & $\begin{array}{l}\text { Em fase de implantação de vários protocolos, da } \\
\text { recepção do hospital até sua alta, visando a } \\
\text { segurança do paciente, principalmente, relacionadas } \\
\text { a cirurgia segura e notificação a eventos adversos. Se } \\
\text { ocorrer será rapidamente rastreado, analisado e } \\
\text { executado o tratamento adequado. }\end{array}$ \\
\hline 16 & $\begin{array}{l}\text { Segurança do paciente } \\
\text { na visão de enfermeiro: } \\
\text { uma questão } \\
\text { multiprofissional }\end{array}$ & $\begin{array}{l}\text { ARAUJO } \\
\text { MAM et al. }\end{array}$ & 2017 & $\begin{array}{l}\text { Rev. } \\
\text { Enfermagem } \\
\text { oline }\end{array}$ & MEDLINE & $\begin{array}{l}\text { Abordagem } \\
\text { qualitativa }\end{array}$ & $\begin{array}{l}\text { A equipe de enfermagem necessita empenhar-se na } \\
\text { promoção da segurança do paciente. E quem é apto } \\
\text { a identificar riscos eminentes é o enfermeiro. }\end{array}$ \\
\hline 17 & $\begin{array}{l}\text { Processo de } \\
\text { enfermagem: } \\
\text { Implicações para a } \\
\text { segurança do paciente } \\
\text { em centro cirúrgico }\end{array}$ & $\begin{array}{l}\text { RIEGEL F, } \\
\text { JUNIOR } \\
\text { NO. }\end{array}$ & 2017 & $\begin{array}{l}\text { Rev. } \\
\text { Cogitare } \\
\text { enfermagem }\end{array}$ & MEDLINE & $\begin{array}{l}\text { Abordagem } \\
\text { qualitativa }\end{array}$ & $\begin{array}{l}\text { O processo de enfermagem no centro cirúrgico vem } \\
\text { fortalecer a segurança do paciente de forma } \\
\text { metodológica, proporcionando a enfermagem o cuidar } \\
\text { sistematizado no pré-operatório, perioperatório e pós- } \\
\text { operatório. }\end{array}$ \\
\hline 18 & $\begin{array}{l}\text { Avaliação sobre a } \\
\text { segurança do paciente } \\
\text { durante o procedimento } \\
\text { anestésico-cirúrgico }\end{array}$ & $\begin{array}{l}\text { STUDART } \\
\text { RMB et al. }\end{array}$ & 2017 & $\begin{array}{l}\text { Rev. } \\
\text { Enfermagem } \\
\text { UFPE }\end{array}$ & LILACS & $\begin{array}{l}\text { Estudo } \\
\text { quantitativo }\end{array}$ & $\begin{array}{l}\text { Os impressos estavam fornecendo informações } \\
\text { adequadas e seguras, a equipe trabalhando em } \\
\text { harmonia e qualidade com o uso do checklist, o que } \\
\text { possibilita uma cirurgia segura livres de riscos para o } \\
\text { paciente. }\end{array}$ \\
\hline 19 & $\begin{array}{l}\text { Ações de enfermagem } \\
\text { para a segurança do } \\
\text { paciente em hospitais: } \\
\text { revisão integrativa }\end{array}$ & $\begin{array}{l}\text { SIMAN AG, } \\
\text { et al. }\end{array}$ & 2017 & $\begin{array}{l}\text { Rev. } \\
\text { Enfermagem } \\
\text { UFPE }\end{array}$ & LILACS & $\begin{array}{l}\text { Abordagem } \\
\text { qualitativa }\end{array}$ & $\begin{array}{l}\text { Não foram abordados assuntos relativos à } \\
\text { importância da identificação do paciente, o qual faz } \\
\text { parte do protocolo da segurança do paciente a partir } \\
\text { do momento que o paciente é admitido no hospital. }\end{array}$ \\
\hline
\end{tabular}

Fonte: LOPES TMR, et al., 2018. 


\section{DISCUSSÃO}

\section{Eventos adversos ocorridos em centro cirúrgicos}

Baseado no princípio hipocrático de "não causar danos", a segurança do paciente tem sido mundialmente mencionada, constituindo-se um grande desafio para a organização da saúde. Dentre o processo de cuidar os riscos são iminentes, portanto observa-se grande dano ao paciente-ocorrendo o aumento na permanência de internação, lesão permanente ou até morte-ocorrida decorrentes da falha na assistência à saúde, relacionados à sobrecarga de trabalho, dimensionamento de pessoas inadequado, a carência de capacitação de trabalhadores e comunicação ineficaz (REIS GAX, et al., 2017).

Segundo Figueiredo ML e D'Innocenzo M (2017), para implementar protocolos de segurança do paciente e evitar eventos adversos, é essencial que os enfermeiros evidenciem quais são os principais eventos adversos que ocorrem na unidade, como por exemplo, eventos adversos relacionados à administração de medicamentos; eventos adversos relacionados à vigilância do paciente; eventos adversos relacionados à manutenção da integridade cutânea; eventos adversos relacionados aos recursos materiais e após essa identificar implementar protocolos de segurança em conformidade as necessidades de cada cliente atendido.

Assim, o centro cirúrgico é um local que oferece ao paciente maior risco, ocasionado por exposição de órgãos vitais, vísceras e músculos; assim exigindo um controle rigoroso de microrganismos e evitando o surgimento de infecções. As maiores partes dos eventos adversos são provocadas por falta de supervisão de vários elementos importantes como, identificação do paciente, se foram removidos próteses, lentes, adornos, realizado demarcação cirúrgica, exames e pré-operatório. Ainda, o paciente comumente está sedado e não pode se comunicar. Além disso, há um grande número de dispositivos tecnológicos que estão ligados ao paciente (LELIS LS, et al., 2017).

Os eventos adversos são considerados como forma simples de identificar erros quantitativamente, são em média $10 \%$ de readmissão hospitalar; portanto a consequência desses eventos adversos é retratada entre o "cuidado real e cuidado ideal". Fato demostrado no relatório do Institute of Medicine, To Err is Human (IOM) que demostra que cerca de 44.000 a 98.000 americanos morrem anualmente em decorrência dos eventos adversos. Considera-se a enfermagem como a maior força de trabalho em saúde, portanto, são os profissionais que passam o maior tempo ao lados pacientes e que necessitam trabalhar ainda mais estratégias para garantir a segurança do paciente, tais estratégias são implantação de protocolos, capacitação de equipe, sistematização da assistência de enfermagem perioperatório (SAEP) e checklist para cirurgia segura (DUARTE SCM, et al., 2015).

\section{Implantação do instrumento de checagem na segurança do paciente (checklist)}

A adoção dos protocolos pela a equipe de enfermagem vem auxiliando a prevenção de ocorrência de eventos adversos, como a não repetição de erros já ocorridos e estimulação de ações corretivas através da adoção de programas de qualificação e capacitação de toda a equipe. Com isso o enfermeiro tem condições de analisar precocemente os riscos-sempre realizando dentro dos aspectos legais e éticos (VELHO JM; TREVISO P, 2013).

Freitas MR, et al. (2014), asseguram que o checklist aplicado corretamente reforça a segurança do paciente no ambiente cirúrgico. Esse instrumento pode ser divido em 3 etapas: antes da indução anestésica, antes da incisão cirúrgica e antes do paciente sair da sala de cirurgia. A aplicação desse instrumento reduz taxas de mortalidade, complicações, números de erros por falha na comunicação da equipe-conforme foi evidenciado nessa revisão.

Assim o checklist é um mecanismo de transformação das práticas cirúrgicas, sendo um sistema capaz de gerenciar a monitorização dos processos continuados, garantindo sua eficácia na segurança do paciente (RIBEIRO HCTC, et al., 2017).

Realização, treinamento, comprometimento e notificação de ocorrência da equipe de enfermagem do centro cirúrgico. 
Para a segurança do paciente ser efetiva tem-se a necessidade de implantar programas de qualificação com atividades educativas, além de investir em espaço com material didático e disponibilidade de tempo o que aumentará a adesão dos profissionais (MIRANDA AP, et al., 2017).

Segundo Gomes LC, et al. (2014), o enfermeiro tem que estar sempre em atualização. A empresa quando oferece novas tecnologias e novos treinamentos aos que trabalham no ambiente do centro cirúrgico, admitem ações integradas para melhorar a assistência ao usuário.

Nesse contexto, as empresas devem buscar a capacitação e o desenvolvimento de seus quadros. Desse modo, um programa de educação voltado aos profissionais de enfermagem requer um planejamento dinâmico, participativo, interdisciplinar e com objetivos definidos, buscando atender diretamente as necessidades da organização e dos profissionais, principalmente o que tange ao profissional do centro cirúrgico (SOUSA CS, et al., 2013).

\section{CONSIDERAÇÕES FINAIS}

Percebeu-se nessa revisão que o aumento da aplicação de protocolos como: SAEP e o checklist no centro cirúrgico foram importantes para garantir a segurança do paciente. Também se percebeu que por meio desses protocolos os eventos adversos começaram a ser identificados e notificados com maior frequência, principalmente pelos enfermeiros. A análise permitiu ressaltar que apesar de ser uma temática bastante discutida atualmente é necessário realizar treinamentos periodicamente e capacitações; com o objetivo de atender aos anseios dos profissionais sobre métodos adequados de conduzir e melhorar a qualidade da assistência prestada-por meio do controle e prevenção dos eventos adversos-com vistas a alcançar melhora na avaliação da qualidade do cuidado.

\section{REFERÊNCIAS}

1. BEZERRA WR, et al. Ocorrência de incidentes em um centro cirúrgico: estudo documental. Rev. Eletr. Enfermagem, 2015; 24-27

2. BIANCHI ERF, LEITE RCBO. O Enfermeiro de centro cirúrgico e suas perspectivas futuras - uma reflexão. Rev. SOBECC, 2016.

3. DUARTE SCM, et al. Eventos adversos e segurança na assistência de enfermagem. Revista brasileira de enfermagem, 2015.

4. FIGUEIREDO ML, D'INNOCENZO M. Eventos adversos relacionados às práticas assistencias: uma revisão integrativa. Enfermagem Global, 2017; 605-650

5. FREITAS MR, et al. Avaliação da adesão ao checklist de cirurgia segura da OMS em cirurgias urológicas e ginecológicas, em dois hospitais de ensino de Natal, Rio Grande do Norte, Brasil. Cadernos de Saúde Pública, 2014; 137-148

6. GOMES JAP, et al. Instrumentos para avaliar a qualidade e segurança no bloco operatório-revisão integrativa. Cogitare enferm, 2016; 01-09

7. GOMES LC, et al. O enfermeiro no gerenciamento do centro cirúrgico. Revista Eletrônica da Faculdade Metodista Granbery, 2014.

8. LELIS LS, et al. Prevenção de eventos adversos relacionados ao procedimento cirúrgico: uma prática da enfermagem. Revista Científica FACMAIS online, 2017; 175-195

9. LIMA AM, et al. Segurança do paciente e montagem de sala operatória: estudo de reflexão. Revista de enfermagem UFPE on line, 2013; 289-294

10. MIRANDA AP, et al. Contribuição da enfermagem à segurança do paciente: revisão integrativa. SANARE-Revista de Políticas Públicas, 2017.

11. OLIVEIRA RM, et al. Estratégias para promover segurança do paciente: da identificação dos riscos às práticas baseadas em evidências. Escola Anna Nery Revista de Enfermagem, 2014; 122-129

12. PANCIERI AP, et al. Checklist de cirurgia segura: análise da segurança e comunicação das equipes de um hospital escola. Revista Gaúcha de Enfermagem, 2013; 71-78

13. REIS GAX, et al. Implantação das estratégias de segurança do paciente: percepções de enfermeiros gestores. Texto \& ContextoEnfermagem, 2017.

14. RIBEIRO HCTC, et al. Adesão ao preenchimento do checklist de segurança cirúrgica. Cadernos de Saúde Pública, 2017.

15. SALBEGO C, et al. Significado do cuidado para enfermagem de centro cirúrgico. Revista da Rede de Enfermagem do Nordeste, $2015 ; 46-53$

16. SECANELL M, et al. A surgical safety checklist implementation: experience of a start-up phase of a collaborative project in hospitals of Catalonia, Spain. Medicina clínica, 2014; 17-24

17. SILVA AH, FOSSÁ MIT. Análise de conteúdo: exemplo de aplicação da técnica para análise de dados qualitativos. Dados em Big Data, 2017; 23-42

18. SILVA JL, et al. Fatores associados à desnutrição em idosos institucionalizados. Revista Brasileira de Geriatria e Gerontologia 2015.

19. SOUSA CS, et al. Avanços no papel do enfermeiro de centro cirúrgico. Revista de enfermagem UFPE on line, 2013; 6288-6293

20. VELHO JM, TREVISO P. Implantação de programa de qualidade e acreditação: contribuições para a segurança do paciente e do trabalhador. Rev. adm., saúde, 2013; 90-94 